\title{
VIDEO-OTOSCOPIC ASSISTED PLASMA ABLATION OF CERUMINOUS CYSTOMATOSIS OF THE EAR OF THE CAT: RETROSPECTIVE STUDY IN 5 CASES
}

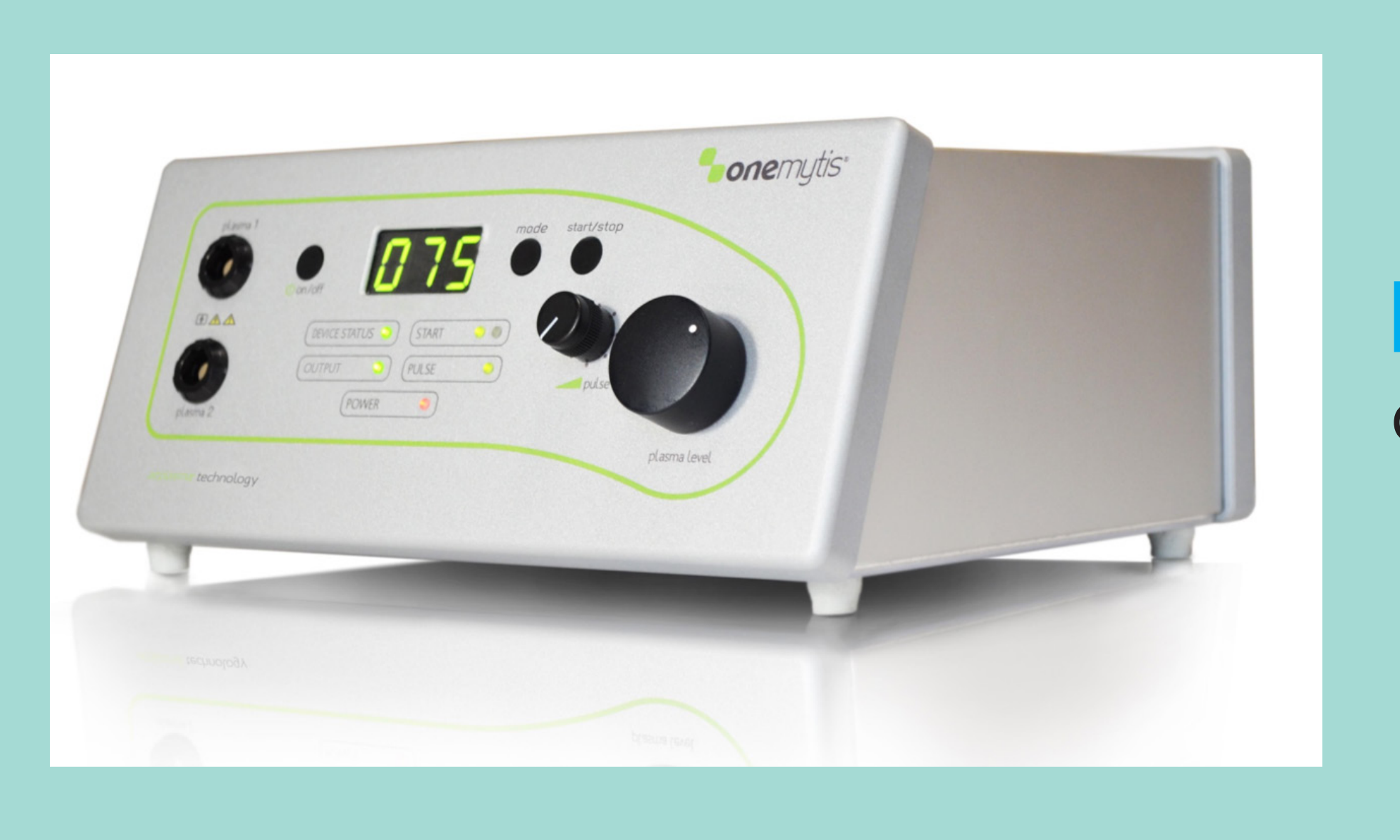

FIG. 1 Onemytis ${ }^{\circledR}$ Air Plasma device

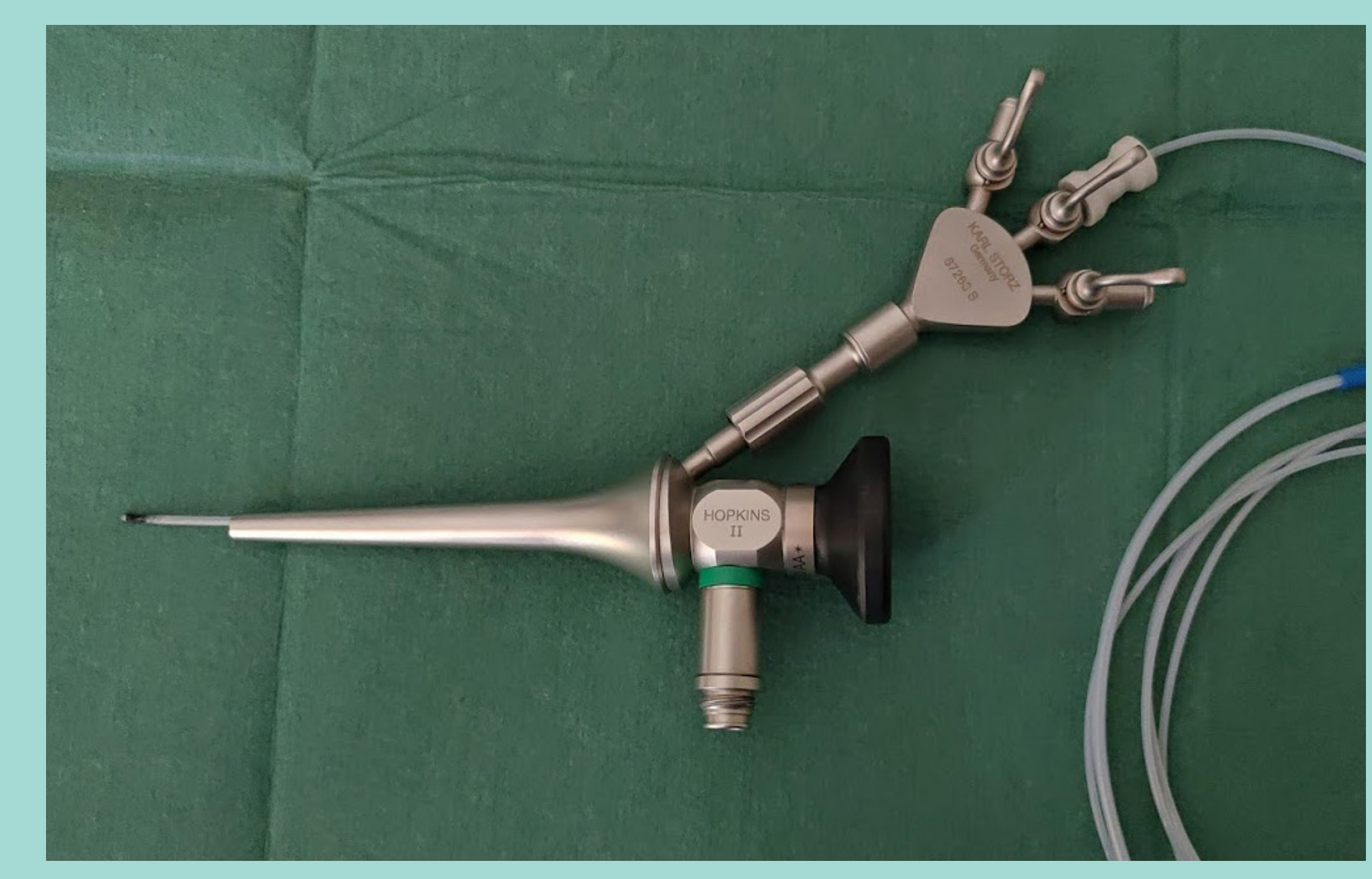

FIG. 2 Otoscope with endoscopic probe for plasma ablation

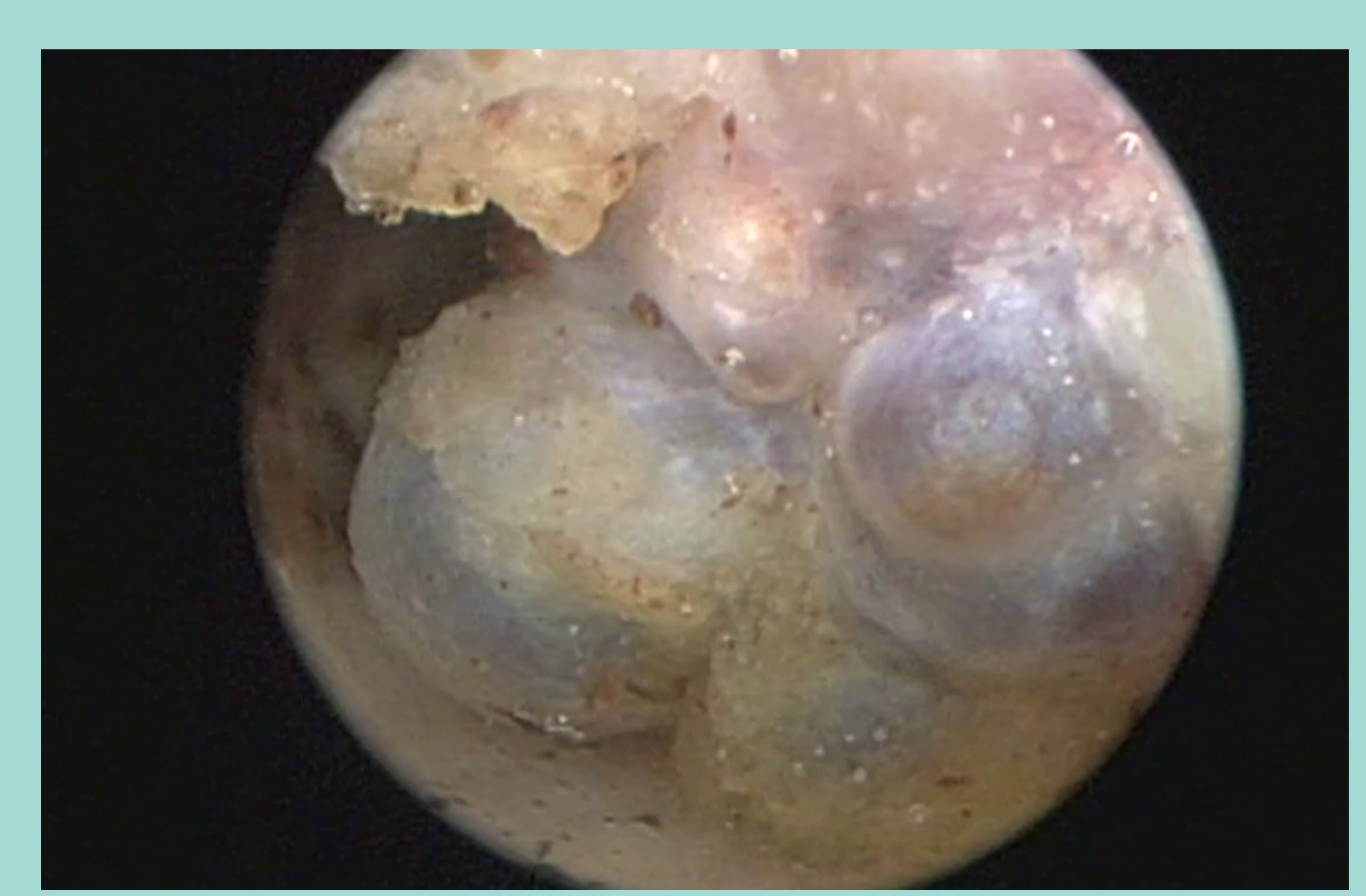

FIG. 3 Multiple dark-blue cystic lesions in the vertical canal

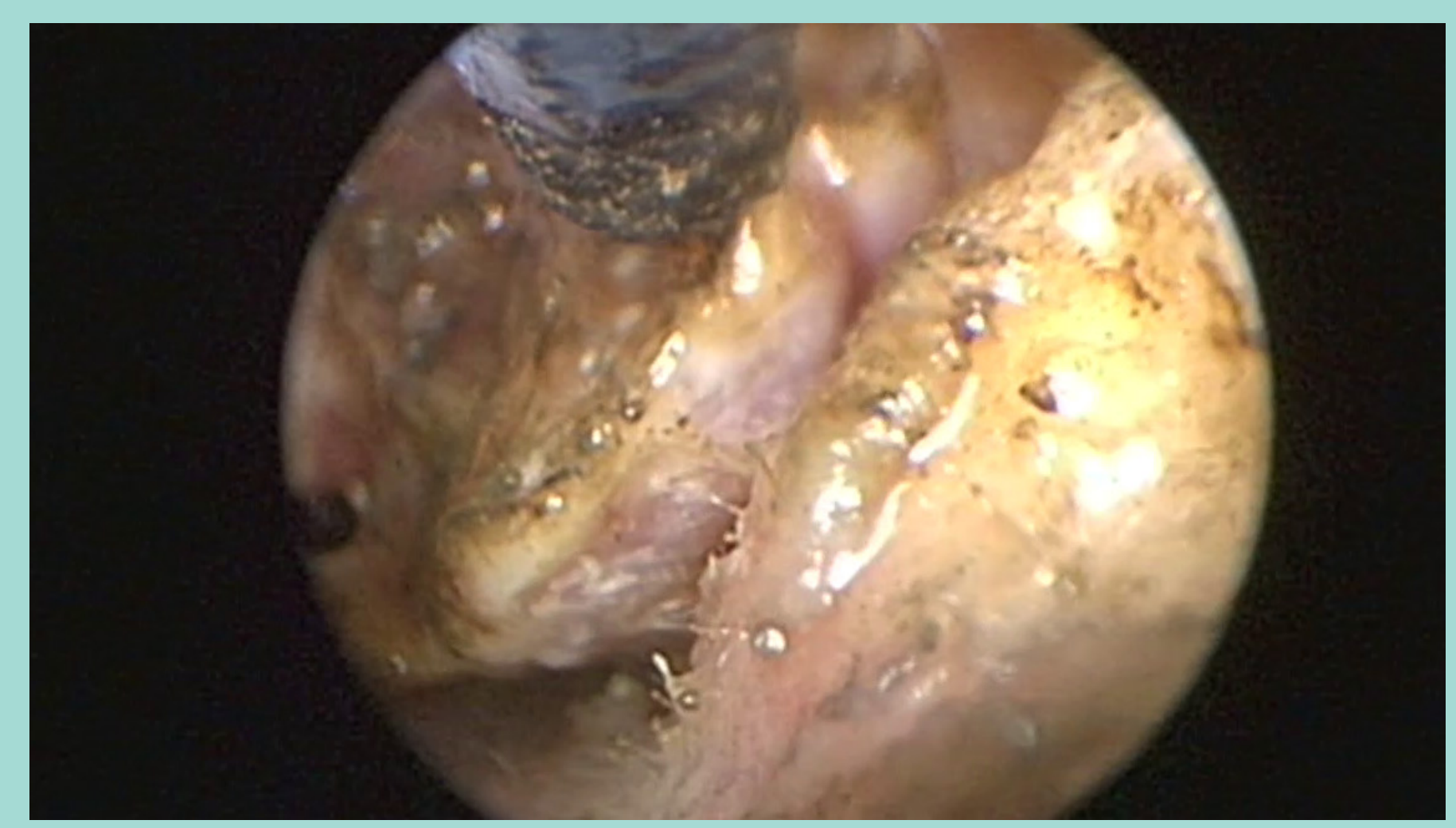

FIG. 4 Video-otoscopic assisted plasma ablation of a cystic lesion in the vertical canal

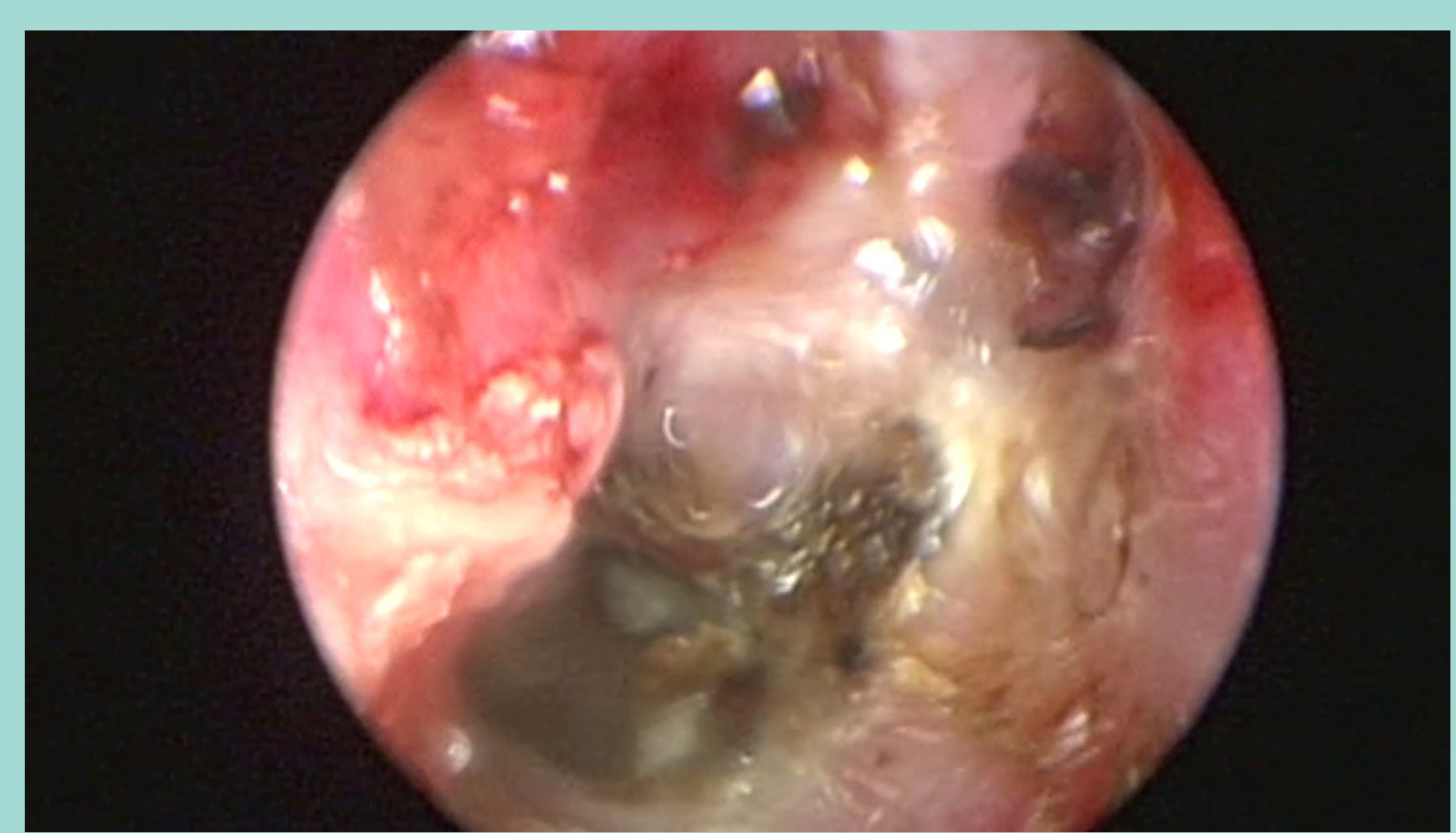

FIG. 57 day post-treatment: marked erythema with brown ceruminous exudate in the vertical canal

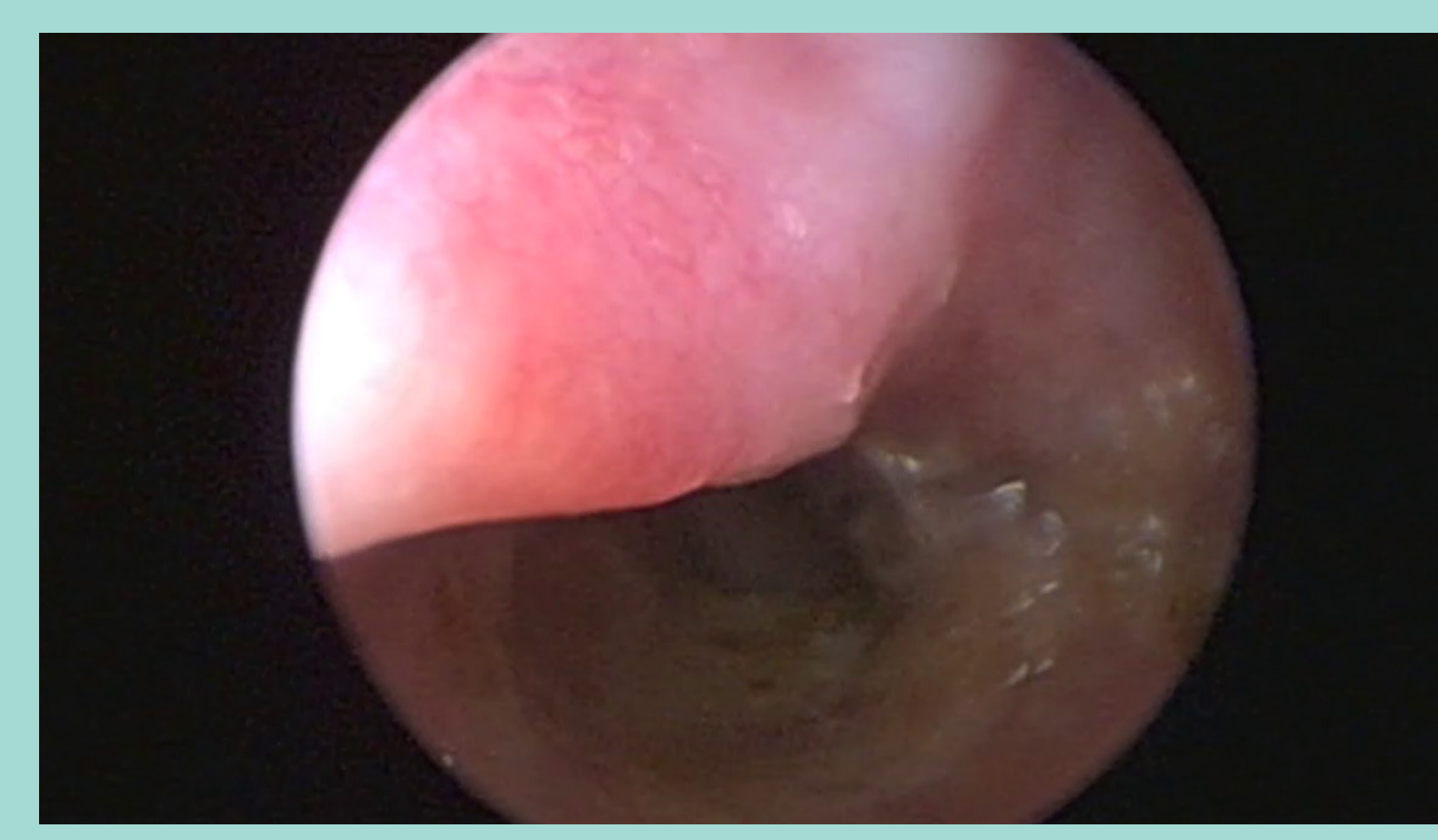

FIG. 660 days post-treatment: normal vertical canal with minimal amount of cerumen
INTRODUCTION: Ceruminous cystomatosis (CC), also known as ceruminous gland hyperplasia/adenoma or apocrine cystoadenomatosis, is a benign skin disorder of the feline ear, of uncertain origin. Macroscopically cystomatosis is characterised by multiple dark blue or brown nodules, usually less than 2 $\mathrm{mm}$ in diameter, located in the external ear canal and vertical canal. Affected cats usually presents signs of obstructive otitis externa, including pruritus, head shaking and othorrea. Diagnosis is based on histological examination of biopsied tissue sampled. Surgery is indicated to remove obstruction by debridement of the ceruminous hyperplasia or by lateral or total ear canal ablation in more severe cases. Carbon dioxide laser ablation of the occlusive nodules is reported to be an effective therapy. An alternative treatment option can be the plasma scalpel, an electrosurgical device that uses pulsed radiofrequency to generate a plasma-mediated discharge along the exposed rim of an insulated blade. Airplasma ${ }^{\circledR}$ is a newly developed technology that makes it possible to generate plasma energy directly from the air, therefore, without using other inert gases (Argon or Helium). The generated plasma is visible in the form of a glow. According to the manufacturer, this device offers the following features: combined cutting, ablation, and coagulation function, reduces invasiveness due to the absence of return plates, necrotized area almost wholly absent or limited, operating temperature less than $50^{\circ} \mathrm{C}$, no need for protection for the operator and patient, and no use of inert gases.

AIM OF THE WORK: The aim of this work is to evaluate the effectiveness of the video otoscopic air plasma ablation of ceruminous cystomatosis in cats.

MATERIALS AND METHODS: Inclusion criteria were histologic diagnosis of CC, a full medical record with radiological and endoscopical imaging and a minimum follow up of six months. Five cats were included.

Of the five cats two were male domestic short hair (DSH), two were female DSH and one was female Persian. Mean age was 91,2 months (range 12-156 months). Signs of chronic otitis externa were seen in all cats.

All cats underwent general anaesthesia.

The integrity of the tympanic membrane was verified with video otoscopic evaluation after a complete cleaning of the ear.

The air plasma generator was connected to a $1.8 \mathrm{~mm}$ endoscopic probe placed through the working channel of the otoscope to perform the ablation.

RESULTS: Re-evaluation one week post surgery showed mild to moderate exudation on the surgical site, with intermittent scratching and head shaking in all cats.

All cats were re-evaluated 14 days post surgery and the surgical site appeared completely healed in all cats. Mild eritema of the pinnae was observed in three cats.

Clinical and otoscopic follow-up examinations at six months after the procedure were normal in all cats

CONCLUSIONS: Air plasma ablation is a safe alternative to laser or surgical ablation of ear's benign neoplasms, with a rapid healing and reduced exposure to volatile organic compounds.

\section{REFERENCES}

Comparative Morphological Effects of Cold-Blade, Electrosurgical, and Plasma Scalpels on Dog Skin, Luca Lacitignola, Salvatore Desantis, Giovanni Izzo, Francesco Staffieri, Roberta Ros- 